\title{
Getting some reality into debates about NBN FTTP
}

\author{
Robin Eckermann \\ University of Canberra
}

\begin{abstract}
Summary. Sentiment about fibre-to-the-premises (FTTP) runs hot in Australia, fuelled by the NBN initiative for Australia's next generation of broadband. Unfortunately most of the debate is ill-informed, focusing on plumbing rather than the uses to which the network may be put. The base of users subscribing to the higher speeds made possible by FTTP is limited right now, and much of their communication will be speed-limited by constraints outside of the NBN FTTP segment. In addition, a growing proportion of Australians choose to operate entirely on mobile connections. For all these reasons, the number of users who would benefit from FTTP in the short term is modest.

The Coalition has proposed greater use of existing infrastructure - in particular, using fibre-to-the-node (FTTN)/DSL technology and potentially hybrid-fibre-coax (HFC) technology - to deliver an upgrade earlier and at lower cost. In the face of cost pressures, it is likely that the original 93\% FTTP target would be adjusted with greater use of wireless and satellite technologies in rural areas and the use of copper for the final link in multi-dwelling complexes. More generally, there is no question that FTTN/DSL solutions can deliver good quality broadband, but further work will be needed to determine where this can be done cost-effectively. Similarly, with appropriate upgrades, HFC networks can deliver next-generation broadband speeds. When practical factors are taken into consideration, the gap between Labor and Coalition plans closes somewhat.

Developing the best strategy for Australia needs to take cost into consideration, and there is scope for NBN Co to improve its performance in this area. Any major cost blowout would have significant ramifications for broadband users and use in Australia. If the rollout of FTTP is scaled back, users with a need may still get access on a user-pays principle - though the practicality of this has yet to be demonstrated. Widespread FTTP remains the right long-term goal for Australia, but the approach for getting there needs to be finessed.
\end{abstract}

\section{Introduction}

Barely a day goes by without encountering passionate views about the role of the National Broadband Network (NBN) in Australia's broadband future. The fervour with which some folk have embraced fibre-to-the-premises (FTTP) as their new religion is reminiscent of that exhibited by enthusiasts of the early Apple Macintosh computers - lugging them around in backpacks, preaching relentlessly to infidels and so on ... but all too often not actually doing much productive with them! Unfortunately, much of the debate about FTTP is ill-informed, and this does not help the cause of advancing Australia's network infrastructure as quickly as possible, and in an efficient and cost-effective manner. 
This paper seeks to contribute to a more balanced discussion by outlining some of the considerations that are all too often neglected amidst the high emotions and politics.

\section{The NBN: a sound vision}

The former Government can rightly claim to have put forward a bold vision for Australia's next generation telecommunications infrastructure. The commercial incentives did not exist for either the incumbent or new market entrants to launch into a significant, wide-scale investment program to upgrade Australia's broadband capacity and, without some form of intervention, Australia risked slipping further behind international leaders.

Further, FTTP is the best available access network architecture today, and there are no signs of an investment in fibre facing the risk of obsolescence any time soon. It is my hope that present and future Governments will recognise the strategic value of extending fibre as close to the customer as possible (ideally to their premises) and work towards this as the right long-term goal in their leadership of the communications portfolio.

Infrastructure-based competition works in some areas of the market - for example, in central business districts and in pockets where there is a concentration of high-value business. For customers in these areas, competition drives efficiency and brings benefits and innovation. There is merit in exposing NBN Co to such competition, rather than protecting it from such healthy forces.

However, in other segments of the telecommunications market where there is not enough customer spending to support multiple networks, natural monopolies are the most efficient approach. Duplicating infrastructure in these areas makes no more sense that building a second set of power lines to give choice as to how power is delivered. The chapter of telecommunications policy that sought to promote facilities-based competition in access networks right across Australia was folly, and it is pleasing to have progressed beyond this.

However, monopolies are ripe for exploitation, so regulatory scrutiny (with the option of intervention) is important.

Monopolies that are run by vertically integrated businesses often result in competition in other "layers" of the value chain being stifled. Accordingly some form of open access framework is appropriate. This was one of the pioneering principles in creating TransACT as an advanced broadband network capable of supporting many different services and service providers. Whilst considered radical some 15 years ago, it is now widely accepted as the new telecommunications wisdom around the world, and it is pleasing to see these principles reflected in the NBN vision. In this respect, the NBN initiative is driving invaluable progress towards the structural separation of access and services. 
Another commendable pillar of the NBN vision is commitment to improving access speeds in rural and regional areas, coupled with uniform wholesale pricing across the country. Uniform wholesale pricing does not guarantee full equivalence of either retail pricing or service levels, but it is certainly a step in the right direction in terms of putting non-urban Australians on a more equitable footing with their city cousins.

\section{What's the problem then?}

What is sadly lacking is an informed public debate of how and how quickly we need to progress to the FTTP end-goal, informed by a better understanding of the role of local access and a realistic assessment of the early performance of NBN Co, the vehicle established to build the national broadband network.

Many supporters of the original NBN plan consider that 93\% of Australians should get FTTP as soon as possible, irrespective of the cost. They understand that broadband is a key economic enabler, but fail to appreciate that FTTP is not necessarily the best way to deliver it in all situations. Even the original NBN vision and current plan recognises this with the use of wireless and satellite delivery technologies where running fibre to all premises would be totally uneconomic.

Public pro-FTTP sentiment has been fuelled by a multi-million dollar advertising campaign, with support for FTTP now extending into quarters of the community who have almost no concept of what it really means! I know this because of the many questions that friends without a technical background have put to me. These folk have got the message the FTTP is the ultimate and they want it, without necessarily understanding why, or considering consequences or alternatives.

In terms of alternatives, journalists who are either politically motivated or ill-informed have promoted the idea that the copper network has almost completely rotted away (it may oxidise, but it doesn't rot!), and that anything less than FTTP would condemn Australians to falling far behind the rest of the world in its exploitation of the digital economy. They take delight in raising the spectre of tens of thousands of ugly green "fridge sized" cabinets littering the kerbs.

The lack of mature understanding about the issues is most recently reflected in a petition (signed by over a quarter of a million Australians!) appealing for the new Government to hold to the original plan to deliver FTTP to 93\% of Australian premises.

I have little doubt that, given a liberally-funded advertising campaign, public sentiment could be similarly whipped up over any number of emotionally appealing issues. For example, a program to upgrade all Australian highways to separated dual-lane carriageways 
would facilitate faster travel, reduce accidents, cut pollution etc. Who wouldn't support this? Never mind that only a tiny minority of Australians have vehicles capable of driving safely at the highest speeds such a road network could support, or that some roads may only be used by a handful of vehicles each week. Never mind that the cost would ultimately draw from the same bucket that funds other worthy priorities such as health, education and social security or that if policy changed to a "user pays" principle, hefty tolls may make the new highways unaffordable to many road users.

An access networks (the final kilometres between central equipment locations and the enduser) is the most expensive part of the network to upgrade because it needs to reach into so many widely-distributed locations. As such, any plan to upgrade it represents a massive investment that should be both planned and implemented carefully. Pressure to drive the program for political gain is a recipe for "more haste, less speed" and for making costly errors that could ultimately come haunt Australians for many years to come.

\section{Getting the perspective right - applications and services}

To achieve a more mature discussion of Australia's next generation of broadband infrastructure, the proper starting point is a consideration of the uses to which the network will be put. Although this article talks much about network plumbing, the infrastructure is really only relevant to the extent that it impedes or enables the applications and services that are available and for which Australians will pay (in initial and ongoing costs).

Because the Internet is a world-wide network, many (but not all) of these applications and services are global in nature. As such, they are designed to use the broadband speeds that are available to a critical mass of users. The number of premises connected at speeds typically achievable only on FTTP networks remains dwarfed by those connected with lowerspeed technologies - notably copper-based DSL, coax-based HFC and wireless. Obviously there is little to be gained commercially by building a product that relies on a target market that has yet to be established.

There are, of course, some generic applications which benefit from raw speed. For example, the use of cloud-based storage is growing, and this commonly requires transferring large volumes of information to or from "the cloud". Similarly, virtual private network (VPN) connections that allow remote users to connect into an employer's office network with full access to shared drives and other resources benefit from maximum speed.

The discussion of "application readiness" should not be limited to bandwidth requirements and availability. In many cases, there are other limiting factors that dictate the rate of progress. By way of two examples only: 
(a) The notion of video-conferencing for a medical consultation has intuitive appeal, but the whole medical sector will need to undergo major change to support this. Doctors need training and equipment. Progress needs to be made in the interoperability of different video-conferencing systems. Established processes and procedures need to be reinvented to handle virtual appointments rather than a queue of patients in the waiting room. Further progress towards electronic patient records is needed, and billing, charging, claims and reimbursement processes need to be revised.

(b) Many employment roles would lend themselves to tele-working some days a week, but in most government agencies and larger corporations, the culture remains quite hostile to change. New measures of productivity are needed. Trust in employees that are "out of sight" needs to grow. Any barriers to equal promotional opportunities for teleworkers need to be dismantled. Corporate information needs to be accessible outside the walls of the central office whilst ensuring that security and privacy responsibilities are upheld. The whole culture needs to change.

It is fair to say that if the bulk of Australians were connected at higher last-mile speed, more local applications and services would undoubtedly develop - and this is one of the legitimate areas in which cost justification for accelerated investment in an FTTP-rich network might be sought.

It is not easy to predict just what form these local applications and services might eventually take. They may be encapsulated in specific products, or they may well be "soft" opportunities - for example, accelerating the pace of innovation and capturing new synergies through closer electronic communication and collaboration between parties in different locations. Whilst it is not possible to foresee the future in such areas, only the brave or foolish would suggest that our "need for speed" will for all times be capped at any particular level. The long-term relevance of high-speed broadband deserves recognition and, based on the state of communications technology as it is known today, widespread FTTP is a sensible long-term goal towards which to work.

\section{User-connection speeds in practice}

Whilst FTTP technology can for all practical purposes abolish last-mile speed limits, this does not per se ensure that all users will have high-speed connections available. Many FTTP-connected users opt for speed-limited services where they are priced lower than premium, high-speed services. This will affect the pace at which a customer-base of nextgeneration broadband users grows, even where FTTP has been deployed. NBN Co's own 
forecasts anticipate a significant proportion of users subscribing to connection speeds that would be easily deliverable without FTTP.

The NBN business model forecasts an increase in its revenue-per-user over time. This projection stands in contrast to historical trends for technology-related goods and services where either costs fall over time, or where users get more for the same cost. Only time could tell how realistic NBN Co's forecast for revenue growth is. If costs do increase as significantly as forecast, broadband may become less affordable to a sector of the population, fuelling rather than alleviating the digital divide.

Rising wholesale costs may also encourage retail service providers (RSPs) to "skimp" on backhaul capacity as their margins are squeezed - eroding may of the benefits of FTTP.

These factors are not intended to deny the nexus between applications/services and infrastructure. Without applications and services, there is little demand (and willingness to pay) for higher speed connections. And without a solid base of users with high-speed connections, there is insufficient demand for next-generation applications and services.

It is difficult to factor as-yet-unidentified future applications and services into a stringent cost-benefit analysis. To some extent, long-term infrastructure investment will always involve strategic decisions that cannot necessarily be defended by guaranteed, measurable returns and future benefits. However, the difficulties of predicting the future should not be seized as a licence for proceeding without regard to measurable costs and benefits.

\section{Understanding that FTTP only turbo-charges the final kilometres of a broadband connection}

Australia's remoteness led Geoffrey Blainey to coin the phrase "the tyranny of distance" in 1966. Originally referring to distance from colonial forebears in Europe, the phase is now commonly used more generally to reflect the socio-economic disadvantages those living in remote areas face in terms of their access to facilities and services, participation in communities and the like. Broadband is one of the developments that can help to break this down. However, when it comes to the Internet, Australia is not an island!

Much of the traffic that users generate is global in nature. As such, it starts on a local access link, traverses Australia's national "trunk" networks, then crosses the oceans over transnational links, enters the national trunk networks of a destination country and finally completes the connection to the destination over foreign access links. The NBN only affects the first few kilometres (and the last few kilometres if it is a domestic connection), leaving everything in-between largely unchanged! 
Already some of the early FTTP users have discovered the reality that paying for the topspeed NBN connection certainly does not guarantee that all of their traffic will perform at lightning speed. Their individual communication flows quickly feed into circuits that carry the aggregated traffic of many users, often with contention ratios like 100:1. FTTP can be a bit like an 8-lane local street that feeds into thin and congested arterial roads and national highways. The overall trip time isn't automatically reduced by having super-fast local streets.

I am fortunate to be connected by a $40 \mathrm{Mbps}$ (downstream)/4 Mbps (upstream) VDSL2 service over copper, and recently had occasion to download two 4 GB images from different overseas sources. The first transfer completed in a little over 15 minutes, operating at the full speed of my local connection. The second took nearly 15 hours! Same high-speed broadband connection, but a very different outcome due to limitations unrelated to that connection.

Many of those supporting the lobby for pervasive FTTP as soon as possible simply don't understand this reality. They naively believe politically-inspired marketing campaigns that suggest the NBN will turbo-charge everything they do, unlock speeds of $100 \mathrm{Mbps}$ and transform their lives with new applications and services. They have the mistaken impression that many next-generation applications (telemedicine etc) simply can't be supported on anything other than FTTP.

The 100 Mbps figure is not especially relevant on multiple counts:

- it is not the connection speed to which the majority of users will subscribe;

- those who do subscribe to a $100 \mathrm{Mbps}$ will commonly experience lower speeds due to other bottlenecks and constraints;

- in any event, it is not the limit of an FTTP network; access speeds of a Gbps (10x faster) are available today, and these will increase further as improved electronics become available over time.

In this regard, let me state again that FTTP represents the current ultimate in bandwidth future proofing. Once the fibre is in place, last-mile speed upgrades can be accomplished simply by configuration changes or (at most) by upgrading electronics. This is why the most widespread possible FTTP is the right end-goal for Australia's telecommunications infrastructure.

However, a "spare no expenses, waste no time" approach to getting to this point will not necessarily unlock commensurate benefits in the short and medium term. 


\section{The growth of mobile dependence}

When considering the growing dependence of end users on broadband connectivity, it is important to acknowledge the role of mobile connectivity. Armed with smart phones, personal digital assistants, tablets, notebooks and other such devices, many Australians are becoming dependent on access to vital information at any time and from any location. Furthermore, this burgeoning market of users is inspiring a generation of mobile applications that in turn fuels the spiralling growth cycle that is the hallmark of the era of the "smart phone" and its relatives.

Mobile connectivity has proven so compelling that for a non-trivial proportion of Australians (estimated at about 15\%), a fixed line broadband connection is no longer deemed necessary.

Greater availability of fibre in urban areas certainly has the potential to improve mobile network capacity by facilitating more smaller cells, and possibly also by allowing more traffic to be off-loaded onto WiFi services.

However, the NBN will do little if anything to directly improve mobile coverage in regional areas. If NBN Co can offer lower cost backhaul from mobile towers, it may marginally improve the business case for the mobile operators to extend coverage at the margins of their network. However, it will not solve the more fundamental problem that many regional Australians face with little or no coverage in areas of low population density.

Limited mobility may be supported with WiFi hotspots surrounding fixed NBN connections, but this is of little value in rural areas because of the distances involved.

It is unrealistic to expect nation-wide geographic coverage for mobile services. However, the absence of coverage along many significant national highways (and for the many small communities living in the corridors surrounding those highways) has very real social and economic consequences for those living in or travelling through regional Australia.

One perspective of the NBN is that it was a response to the market failure to deliver adequate technical progress and competition to Australia's fixed-line infrastructure. A parallel example of market failure exists with mobile coverage and this is unlikely to be remedied by natural market forces. The business case for one operator (let alone three) simply does not work when traffic falls below certain thresholds. The Coalition's commitment to a program for improving regional coverage is a welcome development, and the NBN's relevance could be boosted if it was able to contribute more directly to improved regional outcomes. 


\section{Estimating the users who will benefit from FTTP in the short-term}

In the light of the foregoing, the base of users to whom FTTP will deliver the full potential of the technology in the short term is diminished in a number of ways:

- the $7 \%$ or so who for cost reasons are destined to be served by wireless or satellite technology under the policies of both major political parties;

- the $15 \%$ or so who decline to subscribe to any fixed line service in favour of operating totally on mobile network technology;

- the $50 \%$ or so who will only subscribe to the lowest-rate broadband (12 Mbps downstream and $1 \mathrm{Mbps}$ upstream), many of whom could get a comparable level of performance on existing copper, and certainly on an improved copper network;

- the vast majority of the remainder whose use of applications and services will be constrained by limitations in Australia's national infrastructure, international links and the infrastructure in other countries.

In these circumstances, and whilst not debating the end-goal, it is legitimate to question whether rebuilding Australia's last-mile access infrastructure should be a "waste no time, spare no expense" priority!

\section{A nominal comparison of Labor and Coalition plans}

Figure 1 below compares (prima facie) Labor's nominal plan for a FTTP-rich NBN (A) with the Coalition's alternative making more extensive use of existing network infrastructure (B).

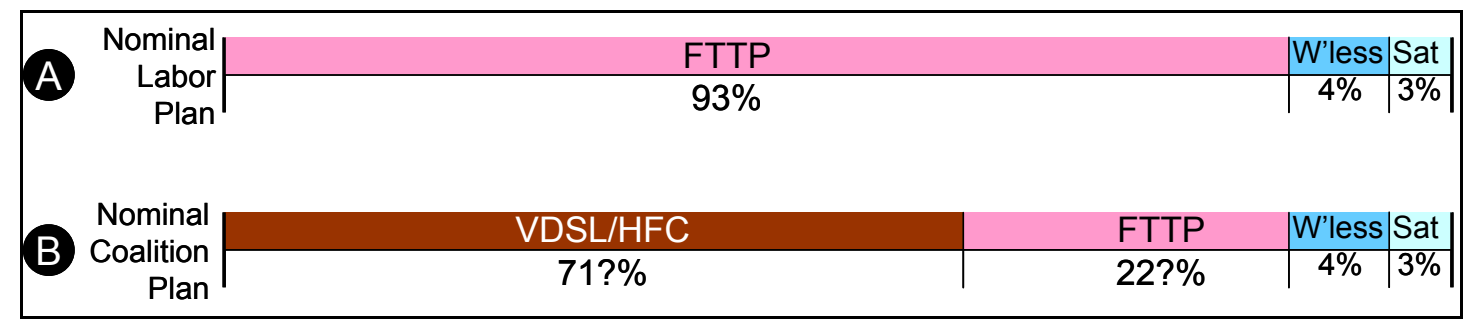

Figure 1. A Nominal Comparison of Labor and Coalition plans

The first thing to note is that both parties propose the same use of wireless and satellite technology for delivering services to the $7 \%$ of Australians living outside of the more densely populated areas (10\% in the original NBN announcement). In a country as large as Australia, it is simply a reality that extending FTTP infrastructure to every last user location would be cost-prohibitive. 
The major difference is the nominal split between FTTP and other access technologies (typically ADSL2+ or VDSL2 over existing copper cabling, or HFC over existing coax cabling). Under Labor, NBN Co's mandate has been to take FTTP to the remaining 93\% of Australian premises. Under the Coalition, the use of existing infrastructure (albeit beefed up for higher performance) may see only an estimated 22\% of Australian premises being connected by optical fibre - most of these in greenfield areas.

\section{Realistic expectations around satellite and fixed wireless connectivity}

Irrespective of the colour of Government, the final $7 \%$ probably needs to be viewed as a little fluid.

Using satellite technology, coverage of the entire Australian continent is achievable, and reasonable speeds can be achieved by managing transponder capacity on a more conservative basis than is typically done with commercially-operated satellite services. However, satellite communications suffer significant delay (latency) due to the long transmission path when a wireless signal is transmitted via a satellite in a geostationary orbit at an altitude of $36,000 \mathrm{~km}$. This can have a debilitating effect on any applications involving real-time communications (telephony, video-conferencing, real-time control, gaming etc). As such, satellite is the delivery technology of last resort - to be used for those premises that cannot be economically serviced with any higher-performing solution.

Wireless connections can support a quality of service on a par with a cabled connection, though typically not to the same peak speeds. However, exact coverage can only be determined having regard to surrounding topology after the base station (tower) locations have been determined. As such, the extent of coverage will only be determined as and when detailed planning takes place. It will also be affected by the locations that are available for deploying wireless base stations. Already some communities have reacted against the deployment of new towers, and there have also been moves to significantly increase site rental costs on Crown land in some jurisdictions. These developments may adversely affect the eventual extent of wireless coverage.

In areas of low population density, wireless coverage can be established more cost effectively than cabled connections. If the costs of upgrading cabled services blow out, it may be tempting to shift the dividing line between the proportion connected by some form of cabling, and the balance connected with wireless or satellite technology. 


\section{Realistic expectations around multi-dwelling units}

NBN Co has encountered difficulty, delay and cost in extending FTTP connections all the way to apartments in multi-dwelling complexes. However, given the relatively short distances from a communications room (commonly) in the basement, the use of VDSL2 technology over in-building copper wiring can support speeds that for the vast majority of users are indistinguishable from those that they might experience with FTTP. This is an approach that NBN Co itself is reported to have considered.

Had the previous Government been returned at the election, this is likely to have been one of the first rationalisations of the "93\% FTTP" plan in the face of cost and time pressures. Approximately $10 \%$ of established Australian residences are multi-dwelling complexes and a further $10 \%$ are town-houses or other higher density forms of construction. Accordingly, it is likely that the $93 \%$ FTTP target would have been moderated by $10-15 \%$ for these premises.

\section{Realistic expectations around the use of existing copper}

Broadband performance over copper is a function of various factors including distance (primarily), gauge (thickness) of the copper, the condition of the cabling and interference (crosstalk) generated by other broadband services in the same cable. On good quality cabling, speeds of $100 \mathrm{Mbps}$ are achievable, with higher speeds possible through the use of vectoring (an interference mitigation technology) and bonding (the use of more than one copper pair).

I am fortunate to be on a VDSL2 service on TransACT's network FTTN/FTTK, operating at 40/4 Mbps over a distance of approximately $1.1 \mathrm{~km}$ of two different grades of copper cabling. In none of my online activities does this service currently limit me. There should be no doubt that it is possible to engineer an access network using copper for the final link to deliver speeds at the levels suggested in the Coalition's policy.

The real question is at what cost can the existing copper network be reconfigured to use VDSL technology over shortened copper loops, and what is the condition of the final kilometre ${ }^{\mathrm{i}}$ or so of that copper across Telstra's network? This will be clarified with future investigations, but the approach promises significant savings by avoiding the need to change anything in the customers' premises - an area of the early FTTP deployment that has been especially problematic.

On the cost side, the Coalition Policy offers the assurance that "... where high maintenance costs or the condition of the copper renders FTTN unattractive ... the best alternative is FTTP". It remains to be seen how extensive these non-viable areas are, but the potential exists for the $22 \%$ to grow substantially if the more pessimistic statements about the 
condition of Telstra's copper have any substance. In my view, it is realistic to expect that the dividing line will shift in favour of FTTP over time.

However, this also represents something of an uncertainty that could materially affect Coalition cost estimates. Cabling (and the associated civil works) represents the bulk of the cost of an access network, and the more that NBN Co has to totally replace the cabling under the Coalition's plan, the more the cost of its alternative will approach the same cost as the original Labor plan (see also Section15 for comments on the likely real cost of a 93\% FTTP deployment).

Renegotiating arrangements with Telstra also remains the other major uncertainty - both in terms of time and any financial impact.

\section{Realistic expectations about the use of HFC}

Although evolved from networks to deliver television services, hybrid-fibre-coax (HFC) networks now have powerful broadband capabilities using the latest DOCSIS cable modem technologies. They can be viewed as another form of fibre-to-the-node architecture where the cabling "downstream" of the node is a shared coax cable.

Ideological objections to the idea of sharing the coax medium that makes up the final link to the customer have no place in the discussion - the FTTP technology chosen by NBN Co also uses a shared fibre medium! However, it is relevant to consider the degree of sharing. Most HFC networks have been deployed with each node serving a relatively large community of users. As a result, there can be communal pressure on the capacity of the coax cabling during times of peak demand.

In the same way as the performance of a copper network can be boosted by extending fibre closer to the customer (and hence shortening the copper distance), the performance of a HFC network can be boosted by pushing fibre closer to the customer (and hence reducing the number of customers on a shared coax segment). Peak speeds of $100 \mathrm{Mbps}$ are readily available today, and much higher speeds are possible with the latest technology.

As with the use of copper, major savings can be achieved if there is no need to change the final cabling from the node to the customer's premises, or anything in the customer's premises. 


\section{A more realistic comparison of Labor and Coalition plans}

In the light of the foregoing, a more pragmatic comparison of the likely outcome under different Government policies might be as reflected in the diagram (Figure 2) below. This does not take into consideration any further moderation of Labor's 93\% FTTP target that might come about due to cost pressures (discussed in the next section).



Figure 2. A pragmatic comparison

In order to assess the impact, this view needs to be meshed with the earlier considerations about how many users can truly benefit from FTTP infrastructure in the near term. Only a subset of those in the "grey zone" who miss out on FTTP are likely to be materially disadvantaged.

\section{Introducing the cost dimension}

Many in favour of retaining a short-term 93\% FTTP target argue that the cost of an FTTP outcome is not dramatically higher than the Coalition's alternative, but that it delivers a much better network. They also argue that any interim solution will require further investment to eventually upgrade to FTTP, and that spending more on the copper network is therefore a wasted investment.

Those arguing this case commonly compare the claimed $\$ 37.4$ billion figure for a 93\% FTTP NBN with a $\$ 29.5$ billion figure for the Coalition alternative. This is not an apples-for-apples comparison since the appropriate Coalition figure to compare with $\$ 37.4$ billion is $\$ 20.4$ billion. However, the far bigger question is whether $\$ 37.4$ billion is a realistic figure - or whether the Coalition's claim that a 93\% FTTP NBN may cost $\$ 94$ billion is closer to the mark.

A diligent, engineering investigation of costs-to-date should expose any looming areas of budget creep in the near future. In the interim, though, it is helpful to consider some of the factors that do not bode well for achieving the original budget: 
(a) The original competitive tender for construction services drew 14 responses from experienced companies, and NBN Co rejected all 14 as opportunistic and exploitative because none came close to the figures on which the original budget was set. Did all fourteen experienced companies get it so wrong - or did one inexperienced company attempting to meet a politically set budget get it wrong?

(b) One of the construction companies (Syntheo) has since extricated itself from NBN work after posting significant losses - something that is not sustainable for any commercial organisation. Others continue to work on construction, but many of their subcontractors are complaining that the rates at which they are being compelled to work are not sustainable.

(c) When probing the large number of homes passed-but-not-yet-connected by the NBN, one finds plenty of "difficult connection" horror stories. Instead of the limited time allowed to complete each connection, connections have taken many hours of effort and repeat visits. In many cases, subcontractors on fixed rates are simply leaving these premises in the "too hard" basket.

(d) Problems have emerged with asbestos in Telstra pits, and with conduits filled with mud.

(e) According to reports, some of the early ribbon-fibre connections have experienced high failure rates, necessitating early rework.

(f) There has been significant slippage against the original schedule, with the consequence that revenues to NBN Co will be also delayed.

In addition to these observations about NBN Co's performance, it is easy to find experienced industry personnel who, based on their first-hand knowledge of building telecommunications networks, variously estimate that holding to the original plan would ultimately cost something in the range $\$ 80-\$ 100$ billion. These "gut feel" estimates are not underpinned by huge models, but at the end of the day, experience can count for a lot more than theoretical analysis all-too-often conducted under pressure to come up with the "right" answers.

Finally, building the NBN with 93\% FTTP would be one of the nation's largest ever infrastructure projects, extending over a time period of 12 years or more - from 2009 until (at least) 2021. How many other similarly large projects don't end up costing double (or more) the original budgets? 


\section{Can costs be contained?}

In the event that honest and experienced scrutiny of the NBN track record to date does indicate larger capital costs, there are some things that could be done to try and limit the blowout.

Aspects of the NBN architecture are arguably over-engineered relative to the normal commercial practice of experienced FTTP operators - for example, the depth to which optical fibre ring topologies are taken. Some of this may be attributable to the learning phase of an organisation that has been rapidly pulled together, driven on a political timescale. Given more time to temper theoretical approaches with real-world experience, there is little doubt that NBN Co's performance should improve going forward. Coupled with some design refinements, it should be possible to moderate capital costs relative to the likely consequences of adhering blindly to the initial approaches.

There are also anomalies and other areas amenable to refinement. For example, under current "adequately served" policy, the NBN Co could be required to overbuild some greenfield estates that are already served by FTTP infrastructure. This is surely not a smart outcome. In addition, the development program seems to prioritise some areas that are comparatively well-served whilst many of those in poorly served areas face a long and uncertain wait for any improvement.

However, dealing with any over-engineering and anomalies is fiddling at the fringes. The main scope for significant capital savings is to shift the goal-posts and set a less ambitious objective.

This is in essence the Coalition's approach to delivering broadband improvements earlier and at a lower cost. In any access network, costs rise disproportionately the closer the new infrastructure extends towards the customer's premises. By retaining use of the existing cabling that leads into virtually every building, a significant reduction in capital costs can be achieved, and time scales for making progress can be accelerated.

Even had there not been a change of Government, if the NBN was shown to be tracking to a significant cost blowout, there would be pressure on capital expenditure, and the use of existing cabling may well have extended beyond multi-dwelling structures as suggested previously (see Section 11). 


\section{The consequences of a cost blowout}

There should be no illusions about the impact of cost overruns. They must either be underwritten from the public purse (our taxes!) or they will flow through to increased enduser costs for fixed broadband connectivity. Each option has significant ramifications.

If underwritten from the public purse, one must consider the extent to which the NBN would add to Australia's debt, and how servicing that debt will detract from other priorities in health, education, other infrastructure etc. It is also fair to ask whether the public at large should bear the burden of cost for a network from which, at least in the early years, only a comparatively small proportion of users will derive a major benefit. If early access to a fibre connection is of such value to these users, might it not be fairer that they make a larger contribution to the cost?

If resolved on a user pays principle, the costs of fixed broadband will rise in Australia, and this could exacerbate rather than narrow the digital divide. Some users may decide to give up a fixed line connection and rely solely on mobile network connectivity - but this simply means that the overall burden of the network would fall on a smaller base of users, putting further upward pressure on costs.

\section{Introducing the time dimension}

Time is the other important consideration.

Four years into the original NBN plan, the nominal date for completion has slipped by four years! The number of homes passed has now been downgraded three times. The number of homes actually connected by NBN fibre at mid-2013 stood at around 44,00o, just 0.5\% of the premises ultimately destined to have access to FTTP under the original plan. Clearly the NBN rollout is proving more difficult than originally envisaged, and the original 8-year plan is now a 12-year plan. In the six months from December 2012 to June 2013, brownfield connections were occurring at a rate of about 300 per working day - still just $5 \%$ the 6,000 per day originally projected for the peak of the rollout.

Some of the slippage can be legitimately attributed to one-time ramping up delays and early teething problems. If permitted to continue on the same trajectory, one would hope that greater momentum would be established and the deployment would proceed at a faster pace.

However, some of the delay must inevitably be attributed to under-estimation of the magnitude of the task. Many experienced industry personnel venture the opinion that rebuilding Australia's national telecommunications infrastructure with a FTTP-rich network is realistically more like a 15-20 year project. The pending review of NBN progress-to-date 
may provide information that would result in any timetable for a 93\% FTTP network being extended substantially.

Whatever the timeframe, those scheduled to gain access to FTTP towards the end of the period under the original plan are in an unenviable position! They would be unlikely to receive any interim upgrade to their connections whilst ever the NBN deployment was looming, and this could see them left years behind other Australians and those in other countries.

A general re-evaluation of deployment priorities coupled with strategies for leveraging existing infrastructure could help to bring at least interim improvements to many Australians much earlier. NBN Co is currently investing its limited capacity in overbuilding areas that are served by some of the best cabling (copper and HFC) in Australia - whilst some of the most poorly served areas don't appear on the radar.

Outcomes - especially for regional Australians - could be improved by prioritising the deployment of backhaul capacity into smaller communities, unlocking the potential of existing copper cabling with the introduction of ADSL2+ or VDSL technology and negotiating such arrangements as are needed to put the cost of wholesale DSL services in these areas on par with those in urban areas.

\section{FTTP on a user-pays model}

For those users who genuinely need speeds only achievable on fibre, the Coalition plan contemplates deploying FTTP on a user-pays principle. From a social equity perspective, it may be reasonable to ask why the community at large should subside the cost of an expensive solution that only a minority of high-end users need. Further, if FTTP is so valuable and can be offered for a contribution as low as $\$ 2,500$, the cost should be seen as a bargain relative to other common technology costs (computers, large screens, software etc).

In practice, however, it may prove quite difficult/expensive to support this option.

If only a tiny proportion of users want (and are prepared to pay for) a FTTP connection, the simplest solution would be to extend individual fibre cables from the node to their premises. However, each new user would require an additional cable to be run, and the size of cable bundles could soon grow to become unmanageable.

The alternative would be to provision spare fibres when any fibre connection is run, not knowing if, when and where those additional fibres may be required. This approach risks converging towards a non-trivial fibre build in parallel to existing cabling - an expensive proposition. 
Notwithstanding the need to work through these potential complications, the user pays principle is already operating in one area of FTTP provision. In new development (greenfield) estates, developers pay at least the cost of pit-and-pipe infrastructure, and this is built into the price of house and land packages. As such, new home buyers are contributing to the capital cost of fibre.

\section{Transition from a shorter-term upgrade to the final goal}

At a broader level, if the goal of widespread FTTP is accepted as the right long-term goal, it is fair to ask whether it makes sense to invest in one round of interim upgrades and then to have to go back later, decommission elements of the interim solution and invest further in the long-term solutions. This is likely to be more expensive overall.

Factors that need to be taken into consideration in balancing short- and long-term investment include:

- the timeframe in which upgrade to FTTP might be required - something likely to be linked to the global pace of FTTP migration;

- declines in technology costs and improvements in technology performance over time (including substantially different approached that may emerge - like fibre technologies that deliver each user a dedicated wavelength); as other countries ramp up FTTP deployments, technology costs can be expected to fall significantly;

- new deployment methods and technologies, offset by a generally rising cost of civil works (a major element in any network build);

- the diminishing value of money over time; and

- the economic value of the disadvantages that a proportion of users would face due to lengthy delays in a "FTTP or nothing" approach.

\section{Summary}

The issues are complex, and what is needed is a more informed debate about the most appropriate way to upgrade Australia's telecommunications infrastructure. The debate should be anchored around a balanced view of key principles:

(a) Focusing on the network plumbing is a mistake - rather the debate should be centred on the uses to which advanced broadband can be put.

(b) To capture the potential benefits of advanced broadband in different sectors, effort needs to be invested beyond infrastructure - in people, processes and change management. This will take many years. 
(c) Taking FTTP to as large a proportion of the population as practical is the right end-goal for Australia.

(d) Open access is the right principle for monopoly infrastructure that cannot be economically duplicated, and structural separation is the best way to support it.

(e) Uniform national pricing (recognising that this involves cross-subsidisation) is a valuable means of improving equity of access for all Australians.

(f) The number of users who will get the full benefit of FTTP access in the near term is limited.

(g) In Australia's largest ever infrastructure project, delay should be avoided, but time is ultimately not of the essence and costs are relevant.

(h) However the plan evolves, interim measures are going to be needed to prevent some Australians being left completely behind in their broadband access.

(i) It makes sense to use existing infrastructure where it can be upgraded cost effectively (having regard to the capabilities achievable and both the capital and operational costs).

(j) Worthwhile interim improvements in connectivity for many Australians living in regional areas could be accomplished by extending competitive backhaul to smaller communities and by reviewing the pricing of copper-based services (like wholesale DSL).

(k) The shortcomings in Australia's mobile coverage need continuing attention. Mobile connectivity is increasingly important to all Australians - it is the "other" pillar of the digital economy. For some Australians, it will remain their only form of connectivity.

\section{Endnotes}

i In (traditional) telephony networks, copper cabling radiates out from an exchange in a tree-and-branch topology. To convert to FTTN, the thick copper bundles nearest the exchange are replaced by optical fibre, retaining only the last few hundred metres of cabling leading into the customer's premises. Any problems with degraded segments of copper cabling that would be replaced by fibre anyway are obviously not relevant.

Cite this article as: Eckermann, Robin. 2013. 'Getting some reality into debates about NBN FTTP'. Australian Journal of Telecommunications and the Digital Economy 1 (1): pp.13.1 13.19. DOI: $10.7790 / a j t d e . v 1 n 1.13 \quad$ Available at: http://telsoc.org/journal 